\title{
CMB Anisotropy Due to Filamentary Gas: Power Spectrum and Cosmological Parameter Bias
}

\author{
Meir Shimon, ${ }^{1}$ Sharon Sadeh, ${ }^{1}$ Yoel Rephaeli ${ }^{1,2}$ \\ ${ }^{1}$ School of Physics and Astronomy, Tel Aviv University, Tel Aviv 69978, Israel \\ ${ }^{2}$ Center for Astrophysics and Space Sciences, University of California, San Diego, La Jolla, \\ CA, 92093 \\ E-mail: meirs@wise.tau.ac.il
}

\begin{abstract}
Hot gas in filamentary structures induces CMB aniostropy through the SZ effect. Guided by results from N-body simulations, we model the morphology and gas properties of filamentary gas and determine the power spectrum of the anisotropy. Our treatment suggests that power levels can be an appreciable fraction of the cluster contribution at multipoles $\ell \lesssim$ 1500. Its spatially irregular morphology and larger characteristic angular scales can help to distinguish this SZ signature from that of clusters. In addition to intrinsic interest in this most extended SZ signal as a probe of filaments, its impact on cosmological parameter estimation should also be assessed. We find that filament 'noise' can potentially bias determination of $A_{s}, n_{s}$, and $w$ (the normalization of the primordial power spectrum, the scalar index, and the dark energy equation of state parameter, respectively) by more than the nominal statistical uncertainty in Planck SZ survey data. More generally, when inferred from future optimal cosmic-variance-limited CMB experiments, we find that virtually all parameters will be biased by more than the nominal statistical uncertainty estimated for these next generation CMB experiments.
\end{abstract}

Keywords: cosmological parameters from CMBR, Sunyaev-Zeldovich effect 


\section{Contents}

1 Introduction 1

2 Power Spectrum Calculation 2

2.1 Filament Model and Comptonization Parameter 2

2.2 Temperature Anisotropy 3

2.3 Power Spectrum 4

2.4 Filament Mass Function 5

3 Cosmological Parameter Bias $\quad 6$

$\begin{array}{lll}4 & \text { Results } & 7\end{array}$

$\begin{array}{lll}5 & \text { Discussion } & 9\end{array}$

6 Conclusions 10

\section{Introduction}

As much as $30-40 \%$ of the baryon mass density of the universe is thought to be in a phase of warm gas distributed along large-scale filamentary structures connecting clusters and superclusters of galaxies. These structures are commonly identified in numerical hydrodynamic simulations [1,2]. Estimated temperature and density ranges are $10^{5}<T<10^{7} \mathrm{~K}$ and $4 \cdot 10^{-6}<n<10^{-4} \mathrm{~cm}^{-3}$, respectively [1,3]. Emission from this gas would be expected in the soft X-ray region, yet its detection constitutes a major observational challenge due to the very low surface brightness and confusion by other extended sources such as clusters and groups.

Observational evidence for this so-called warm-hot intergalactic medium (WHIM) is still quite scant, mostly from measurements of absorption lines in the spectra of background AGN. These include e.g. measurement of an O VIII absorption system in the spectrum of a quasar observed in the Virgo cluster region, possibly due to WHIM around the cluster [4], detection of WHIM associated with the Coma cluster by identifying Ne IX and O VIII absorption lines in the spectrum of X Comae, an active galactic nucleus (AGN) located behind the Coma cluster [5], observation of X-ray emission from the WHIM in the Shapley supercluster region [6], in a high Galactic latitude ROSAT field [7], and in the outskirts of the Coma cluster [8], as measured by X-ray Multi-Mirror Mission (XMM-Newton).

Morphological and statistical studies of filamentary dark matter (hereafter DM) structures in N-body simulations were conducted by e.g. [9,10], who addressed such properties as filament lengths, masses, and shapes, the DM density distribution along and across filaments, and the number of filaments per cluster. More recently, Klar \& Mücket [11] have performed hydrodynamical simulations, studying in detail hydro- and thermodynamical properties of intra-filament (IF) gas, including its temporal evolution.

The WHIM may obviously have other detectable signatures, most important of which would be its impact on the spatial structure of the cosmic microwave background (CMB): Compton scattering of this radiation field by free electrons induces an additional component 
to the anisotropy due to the SZ effect in virialized systems, mostly clusters of galaxies. The WHIM-induced contribution could be appreciable even though the gas is cooler and significantly thinner than intracluster (IC) gas, owing to the long path lengths associated with the filamentary WHIM. CMB anisotropy induced by IC gas - the dominant source of (secondary) anisotropy on angular scales of a few arcminutes - has been extensively studied, e.g. [12-14], resulting in detailed predictions of its power spectrum and cluster number counts.

In contrast, the related anisotropy due IF gas has attracted little attention, likely due to its presumed low level, and the scant observational information on properties of IF gas. These were explored by cosmological hydrodynamical simulations, first by Cen \& Ostriker [1]. An analytical study of the power spectrum of the Sunyaev-Zeldovich (SZ) effect in intergalactic gas (IGG) was carried out in [15], who assumed a log-normal random baryon field for the IGG. In this scenario the contribution to SZ power levels is likely to originate in both larger and smaller structures, such as filaments, halos, and possibly even smaller objects. More recently, in their analysis of the Santa Fe Light Cone Simulation, Hallman et al. [2] identified groups and clusters down to $5 \times 10^{13}$ solar masses. Subtracting the SZ signal from these systems, they mapped the residual magnitude of the Comptonization parameter $y$, finding rather high levels, typically a third of the mean level expected in clusters.

In this paper we describe the results of an analytical calculation of the SZ power signature of IF gas on the CMB and its impact on cosmological parameter inference from the CMB. The calculation of the power spectrum consists of a simple morphological modeling of filaments and their cosmic abundance, an approach which is a direct extension of previous work on SZ cluster-induced anisotropy. Given the current limited knowledge of IGG properties and the morphological complexity of gaseous IG structures, we are inevitably compelled to model them in the simplest manner possible, which nonetheless provides us with the flexibility of testing and exploring several inherent limitations associated with cosmological simulations. The calculation of the bias that the filaments-induced CMB anisotropy introduces on cosmological parameter inference is standard and follows the conventional Fisher information matrix methodology.

The structure of this paper is as follows: Following the Introduction, Sec. 1, we detail in Sec. 2 the simple model we adopted for the morphological and physical properties of the IF gas. We further describe the cosmological abundance of filaments, and lay out the computation methodology of the power spectrum. In Sec. 3 we describe the calculation of the cosmological parameters bias. Results of our work are presented in Sec. 4, discussed in Sec. 5, and summarized in Sec. 6 .

\section{Power Spectrum Calculation}

\subsection{Filament Model and Comptonization Parameter}

The complex web of filamentary structure is difficult to model, but for our purposes here we can roughly describe filaments as isolated entities whose main gaseous sections have cylindrical morphology. Using cylindrical coordinates in the frame of the filament, and assuming a uniform IF gas temperature and density, the Comptonization parameter at position $\vec{r}=(r, \phi, z)$ can be expressed in terms of the cross sectional (2D) radius $R$ of the filament, its length along the symmetry axis, $L$, and Heaviside step functions

$$
y(r, \phi, z)=y_{0} \Theta(R-|r|) \Theta(L / 2-|z|),
$$


where $y_{0} \equiv \int \frac{k_{B} T_{e}}{m_{e} c^{2}} \sigma_{T} n_{e} d \ell$, with $T_{e}, n_{e}$, and $\sigma_{T}$ denoting the electron temperature, density and the Thomson cross section, respectively, and $d \ell$ a line element along the line of sight (los); the remaining quantities have their usual meanings. In our numerical calculations we take $T_{e}=1 \mathrm{keV}$ and $n_{e}=4.3 \times 10^{-6} \mathrm{~cm}^{-3}$, but these are just fiducial values gauging their allowed ranges. As in virialized systems, the gas mass fraction is taken to be a constant fraction $f_{g}$ of the total mass, $M$; denoting the hydrogen mass fraction in the gas as $X$, the (total) IF mass density is

$$
\rho_{m}=\frac{n_{e} m_{p}(X+1)}{2 f_{g}},
$$

where $m_{p}$ is the proton mass, and $X=0.59$ (in fully ionized gas). We take $f_{g}=0.12$, a typical value for the cosmological baryon fraction of the mass density, which also proves to be consistent with the assumption that a fraction $30-40 \%$ of the total baryonic matter in the universe is in filaments (as is discussed later in this section). The inferred filament length

$$
L=\frac{M}{\pi R^{2} \rho_{m}}
$$

is expressed in terms of $R$, which is scaled to a value of $1.5 \mathrm{Mpc} \cdot h^{-1}$, typical over a wide range of filament masses [9].

\subsection{Temperature Anisotropy}

We first compute the Comptonization parameter Fourier transform, required for estimating the power spectrum of the temperature anisotropy. With the relations detailed in the previous subsection, the Fourier transformed $y$ in the filament frame can be calculated analytically, writing

$$
\begin{aligned}
& \vec{r}=r \cos \phi \overrightarrow{\hat{x}}+r \sin \phi \overrightarrow{\hat{y}}+z \overrightarrow{\hat{z}} \equiv r \overrightarrow{\hat{n}}+z \overrightarrow{\hat{z}} \\
& \vec{k}=k \cos \phi^{\prime} \overrightarrow{\hat{x}}+k \sin \phi^{\prime} \overrightarrow{\hat{y}}+k_{z} \overrightarrow{\hat{z}} \equiv k \overrightarrow{\hat{n}}+k_{z} \overrightarrow{\hat{z}},
\end{aligned}
$$

so that $e^{i \vec{k} \cdot \vec{r}}=e^{i k r \cos \phi} e^{i k_{z} z}$, where $r=\sqrt{x^{2}+y^{2}}$ and $k=\sqrt{k_{x}^{2}+k_{y}^{2}}$. Consequently,

$$
\begin{aligned}
& \tilde{y}(\vec{k})=\int_{0}^{2 \pi} \int_{-\infty}^{\infty} \int_{0}^{\infty} y_{0} \Theta(R-|r|) \Theta(L / 2-|z|) e^{i k r \cos \phi} e^{i k_{z} z} r d r d z d \phi \\
& =\frac{4 \pi y_{0} R}{k k_{z}} J_{1}(k R) \sin \left(\frac{h k_{z}}{2}\right) .
\end{aligned}
$$

The desired expression is the Fourier transform of $y$ in the observer frame, which is chosen such that the axis of symmetry of the filament forms an angle $\theta$ with the los to the filament, as illustrated in Figure 1. Transforming the components of the wave vector $\vec{k}$ in the filament frame into the observer frame $\overrightarrow{k^{\prime}}$ is easily done as

$$
\begin{aligned}
& k_{x}=k_{x}^{\prime} \cos \theta-k_{z}^{\prime} \sin \theta \\
& k_{y}=k_{y}^{\prime} \\
& k_{z}=k_{x}^{\prime} \sin \theta+k_{z}^{\prime} \cos \theta,
\end{aligned}
$$

where primed and unprimed quantities correspond to the observer and filament frames, respectively. Substituting for the unprimed coordinates in equation (2.5) yields the Fourier 


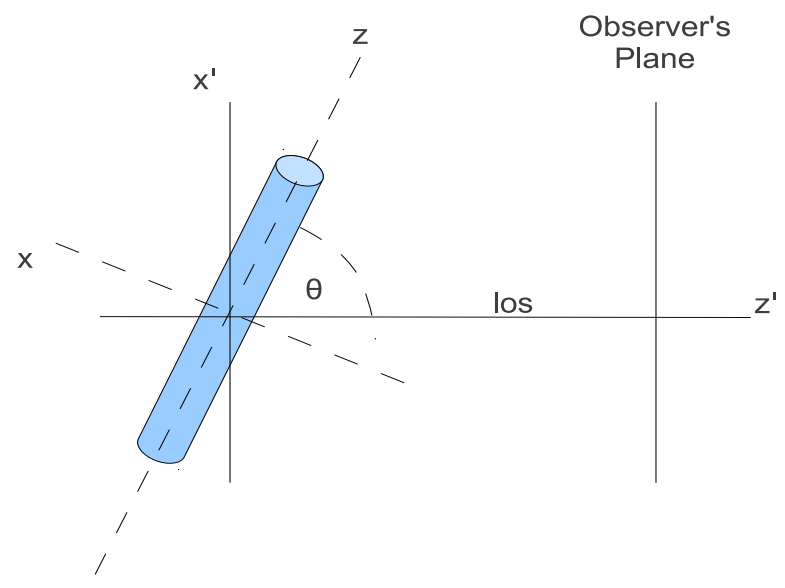

Figure 1. The observer (primed) and filament (unprimed) reference frames. The filament symmetry axis is inclined by an angle $\theta$ to the los (figure adapted from [16]).

transformed profile of the filament y-parameter in terms of the components of the wave vector in the observer frame,

$$
\tilde{y}\left(\overrightarrow{k^{\prime}}\right)=\frac{4 \pi y_{0} R J_{1}\left[R \sqrt{\left(k_{x}^{\prime} \cos \theta-k_{z}^{\prime} \sin \theta\right)^{2}+k_{y}^{\prime 2}}\right] \sin \left[\left(k_{x}^{\prime} \sin \theta+k_{z}^{\prime} \cos \theta\right)(L / 2)\right]}{\sqrt{\left(k_{x}^{\prime} \cos \theta-k_{z}^{\prime} \sin \theta\right)^{2}+k_{y}^{\prime 2}}\left(k_{x}^{\prime} \sin \theta+k_{z}^{\prime} \cos \theta\right)} .
$$

\subsection{Power Spectrum}

The 3-dimensional power spectrum due to a single filament, as would be measured by the observer, can be derived by taking the ensemble average of $y^{2}(k)$. The 3- and 2-dimensional power spectra can be related by expressing the latter as an angular Fourier integral over the projected (angular) profile of the Comptonization parameter, which, in turn, can be evaluated by means of a los integral over the 3 -dimensional y-parameter. Implementing then the flat sky approximation, one arrives at the following expression for the 2-dimensional angular power spectrum [17]:

$$
P_{2}(\vec{l})=\frac{P\left(l / D_{A}\right)}{D_{A}^{2}},
$$

where $D_{A}$ denotes the comoving angular diameter distance, $P\left(k=l / D_{A}\right)$ is the 3 -dimensional power spectrum evaluated at wavenumber $k=l / D_{A}$, and $\vec{l} \equiv\left(l_{x}, l_{y}\right)$ is a two dimensional vector in a flat patch of sky. Global homogeneity and isotropy imply that the power spectrum depends only on the magnitude of the vector $l \equiv \sqrt{l_{x}^{2}+l_{y}^{2}}$. Explicitly,

$$
P\left(l_{x}, l_{y}\right)=\left[\frac{4 \pi y_{0} R J_{1}\left[R \sqrt{\left(l_{x} / D_{A}\right)^{2} \cos ^{2} \theta+\left(l_{y} / D_{A}\right)^{2}}\right] \sin \left[l_{x} \sin \theta(h / 2) / D_{A}\right]}{\sqrt{\left(l_{x} / D_{A}\right)^{2} \cos ^{2} \theta+\left(l_{y} / D_{A}\right)^{2}}\left(l_{x} / D_{A}\right) \sin \theta}\right]^{2} .
$$

In order to find the power spectrum at a given $l$, the contributions from all $\left(l_{x}, l_{y}\right)$ pairs such that $l_{y}=\sqrt{l^{2}-l_{x}^{2}}$ must be summed up. This can be easily carried out by working in polar coordinates, in which $\left(l_{x}, l_{y}\right)=(l \cos \phi, l \sin \phi)$. Since the power spectrum is quadratic in both 
components of $\vec{l}$, it suffices to integrate over the first quadrant of the Cartesian coordinate system and multiply the result by a factor 4 .

The resulting power spectrum has to be convolved with a representative mass function in order to calculate the integrated spectrum of the population of filaments.

\subsection{Filament Mass Function}

Shen et al. [18] derived mass functions for sheets, filaments, and haloes using an excursion set model with a mass-dependent moving barrier. In this formalism, filaments are generated whenever the random walk crosses a first barrier to form a sheet, followed by a second barrier cross, creating the filament. The resulting mass function is described by the fitting formula

$$
\nu f(\nu)=\sqrt{\frac{\nu}{2 \pi}} e^{-\nu\left(1+\beta \nu^{-\alpha}\right)^{2} / 2}\left\{1+\frac{\beta}{\nu^{\alpha}}\left[1-\alpha+\frac{\alpha(\alpha-1)}{2 !}+\cdots\right]\right\},
$$

where $\nu \equiv\left[\delta_{c}(z) / \sigma_{M}\right]^{2}$. Here $\delta_{c}$ and $\sigma_{M}$ denote the usual critical overdensity for spherical collapse and the mass variance at mass scale $M$, respectively. For filaments, Shen et al. provide the fitting parameters $\alpha=0.28$ and $\beta=-0.012$, for which the series within the brackets converges to a value of 0.654 . Consequently, the mass function assumes the form

$$
\nu f(\nu)=\sqrt{\frac{\nu}{2 \pi}} e^{-\nu\left(1-0.012 \nu^{-0.28}\right)^{2} / 2}\left(1-\frac{7.85 \cdot 10^{-3}}{\nu^{0.28}}\right) .
$$

The integrated power spectrum due to a population of filaments can now be computed with the usual expression

$$
C_{\ell}=g^{2}(\nu) \int_{z} \int_{M} d z \frac{d V}{d z} d M \frac{d n(M, z)}{d M} P_{2}(l),
$$

where $g(\nu)$ denotes the spectral distortion of the CMB due to the SZ effect, and

$$
\frac{d n(M, z)}{d M} \equiv f(\nu) \frac{\bar{\rho}}{M} \frac{d \nu}{d M},
$$

where $\bar{\rho}$ denotes the mean background matter density. We integrated the mass function over the redshift and mass ranges of $0.01 \leq z \leq 6$ and $10^{12} M_{\odot} \cdot h^{-1} \leq M \leq 10^{16} M_{\odot} \cdot h^{-1}$, respectively. Note that the total baryonic mass contained in the filament population described by the mass function should constitute a fraction $30-40 \%$ of the total baryonic mass. Integrating the expression $\int n(M, z) M d M$ over the relevant mass range and dividing it by a volume element occupied in a redshift shell $\Delta z$ at $z \approx 0$ provides the total filament mass density in the present universe, whereas its baryonic density can be assessed by multiplying this quantity by the presumed gas fraction $f_{g}=0.12$. The total cosmic baryonic density in the present universe can be evaluated as the product $\Omega_{b} \rho_{c_{0}}$. We verified that the baryonic density contributed by the filament population constitutes indeed a fraction $30-40 \%$ of that implied by $\Omega_{b}$, or specifically $\sim 32 \%$ for $f_{g}=0.12$ and the choice of cosmological parameters detailed in Section 4.

In order to account for all possible filament axis - los configurations, we integrated Eq. (2.12) over the inclination angle range $[0, \pi / 2]$, assigning each angle a weight $\sin (\theta)$. The calculations were carried out in the Rayleigh-Jeans region, for which $g(\nu)=-2$, and for the WMAP 7-year cosmological parameter set, $\Omega_{m}=.266, h=.71, n=.963, \sigma_{8}=.801$, [22]. 
Due to the very steep dependence of the mass function on $\sigma_{8}$, we explored its 1- $\sigma$ uncertainty as well.

Once the temperature anisotropy induced by filaments is calculated - our power spectra are shown in Figure 2 and presented in Section 4 - it is prudent to assess the bias it introduces in cosmological parameter inference, and compare it to the similar cluster-related bias; this is discussed in the next Section.

\section{Cosmological Parameter Bias}

The calculated power spectra shown in Figure 2 indicate that the contribution of filaments to the power spectrum up to $l \approx 1000$ is comparable to that of galaxy clusters. It has been shown in [19] that even after removing clusters detected by the PLANCK SZ survey residual contribution to the power spectrum is sufficiently large to significantly bias the inferred cosmological parameters. Simulated cluster detection and removal that we assume for this work (just for the sake of comparison with the filament-induced bias) is further discussed in the next section.

One could in principle further reduce this bias by removing the contribution of many other clusters not detected by CMB experiments and are detected by other means. For example, the Dark Energy Survey (DES) is expected to detect $\sim 170,000$ galaxy clusters with a mass limit $M_{\min } \approx 5 \times 10^{13} M_{\odot}$ out to redshift $z \sim 1.5$. Assuming that we trust our understanding of the formation of such low mass clusters and that our model of virialized clusters is indeed valid, it could be possible to significantly suppress the contribution of galaxy clusters to the biasing power spectrum to negligible levels. This cannot be done with filaments; individual filaments are hard to detect (also) in the microwave band due to their only mildly nonlinear density contrast. Moreover, unlike clusters these structures contribute at lower multipoles, where the CMB is the dominant signal, further challenging their detection and removal from CMB maps.

Even detection and removal by other means (such as measurement of the extremely low $\mathrm{X}$-ray surface brightness) would be very challenging and obviously uncertain to the level that their removal might induce unwanted systematics in the data. Yet, filaments contribute to the statistical signal, i.e. power spectrum, at a level which might bias cosmological parameter estimation as we show below.

Quantitatively, the statistical uncertainty on a given parameter $\lambda_{i}$ is retrieved from the Fisher information matrix

$$
F_{i j}=\sum_{l} f_{s k y}\left(\frac{2 l+1}{2}\right) \operatorname{Trace}\left[\mathbf{C}^{-1}\left(\partial \mathbf{C}_{l} / \partial \lambda_{i}\right) \mathbf{C}^{-1}\left(\partial \mathbf{C}_{l} / \partial \lambda_{j}\right)\right]
$$

where for each $l$ the symmetric matrix $\mathbf{C}$ is

$$
\mathbf{C}_{l} \equiv\left(\begin{array}{cc}
C_{l}^{T T} & C_{l}^{T E} \\
C_{l}^{T E} & C_{l}^{E E}
\end{array}\right)
$$

The statistical uncertainty on the parameter $\lambda_{i}$ is $\sigma_{\lambda_{i}}=\sqrt{\left((\mathbf{F})^{-1}\right)_{i i}}$. Calculating the bias $\delta_{\lambda_{i}}$ (see, e.g., [19])

$$
\frac{\delta_{\lambda_{i}}}{\sigma_{\lambda_{i}}}=-\sum_{l} f_{s k y} \sigma_{\lambda_{i}}\left(\frac{2 l+1}{2}\right) \operatorname{Trace}\left[\mathbf{C}^{-1}\left(\partial \mathbf{C}_{l} / \partial \lambda_{i}\right) \mathbf{C}^{-1} \Delta \mathbf{C}_{l}\right]
$$



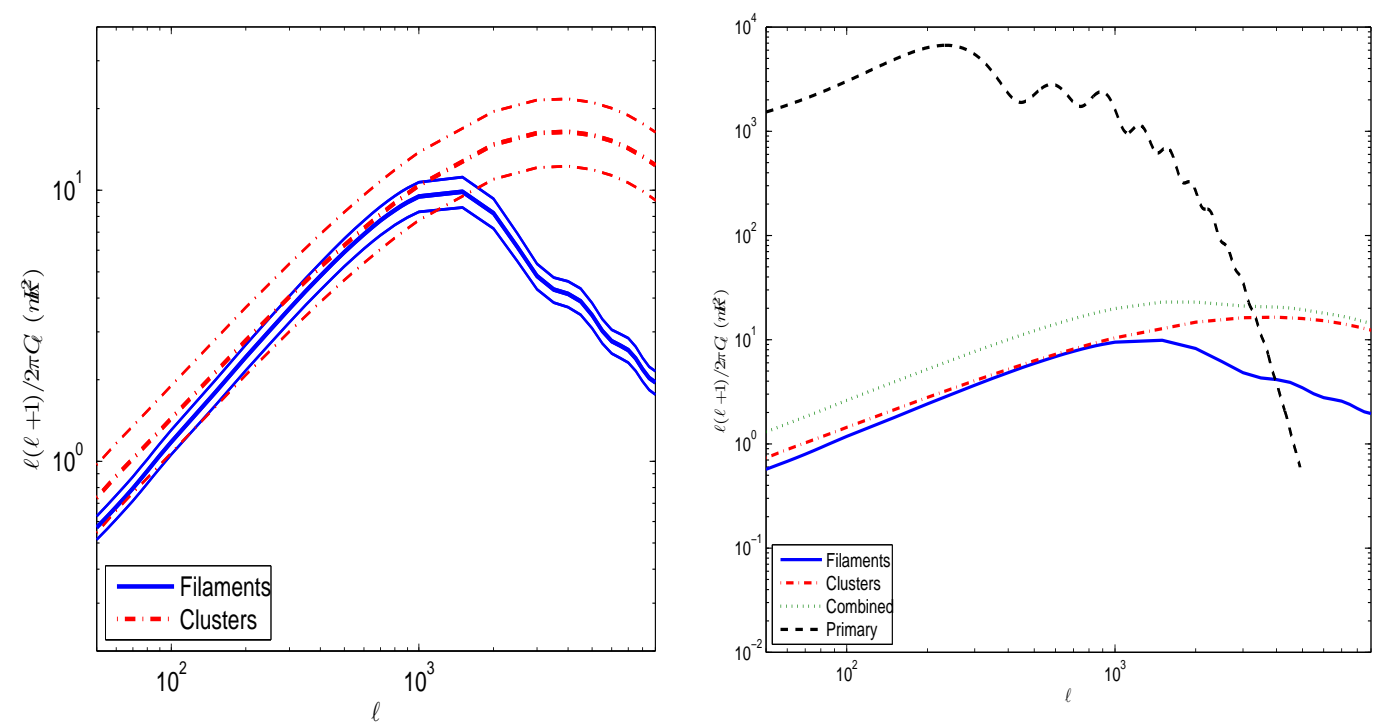

Figure 2. Left panel: SZ power spectra due to filaments (continuous blue), and clusters (dasheddotted red), at the RJ limit. The envelopes mark the $1-\sigma$ uncertainty interval $\sigma_{8}=0.801 \pm .03$, as inferred from the WMAP 7-year results. Right panel: cluster- and filament-induced SZ power plotted together with the primary anisotropy in units of $\mu K^{2}$. The combined SZ power due to clusters and filaments is shown by the dotted green curve.

where here the relative bias $\delta_{\lambda_{i}} / \sigma_{\lambda_{i}}$ is with respect to the nominal uncertainty in the parameter $\lambda_{i}$. Note that only the $(1,1)$ element of the matrix $\Delta \mathbf{C}_{l}$ is non-vanishing; all other elements vanish as no (even statistically) detectable polarization is expected from filaments.

Before we proceed to the results it is important to describe how we actually calculated the cluster and filament contribution to $\Delta \mathbf{C}_{l}$. When maps at different frequency bands below and above the $\mathrm{SZ}$ crossover frequency $(\sim 218 \mathrm{GHz})$ are co-added, there is a partial cancellation of the (dominant, thermal) SZ component. Maps obtained from the various frequency channels are weighted by their inverse (measured) power and linearly combined into a single power spectrum $[20,21]$. The weights are

$$
\left(w_{l}\right)_{i}=\frac{\sum_{j}\left(C_{l}^{-1}\right)_{i j} e_{j}}{\sum_{i, j} e_{i}\left(C_{l}^{-1}\right)_{i j} e_{j}}
$$

where the indices $i$ and $j$ run over all frequencies, the matrix $C^{-1}$ is the inverse total power spectrum, and all components of the vector $e$ are identically 1 . The residual foreground power spectrum used in Eq. (3.3) is then $\Delta \mathbf{C}_{l}=w_{l}^{T} \cdot\left(\mathbf{C}^{-\mathbf{1}}\right)_{l} \cdot w_{l}$.

\section{Results}

Results of the calculation are illustrated in the left-hand panel of Fig. 2, where filamentinduced SZ power levels (marked in blue) are compared with those generated by SZ clusters (red). As expected, filament-induced SZ power peaks at lower multipoles $(\ell \sim 1500)$ as compared with that due to clusters $(\ell \sim 4000)$, owing to the appreciably larger scales involved. With our choice of cosmological parameters and IF gas properties, the filaments-induced 
power is comparable to that of clusters for $\ell \lesssim 1000$. The oscillating pattern seen at multipoles $\ell \gtrsim 3000$ results from the sharp filament edge in our simplified cylindrical model. The combined cluster and filament power plotted in the right-hand panel of the figure suggests that the filament-induced SZ signal may bias cosmological parameter inference from the primary anisotropy (at $\lesssim 1000$ ) at a similar level to that due to clusters.

The procedure for estimation of the statistical uncertainty and bias described in Sec. 3 was applied to our 9-parameter cosmological model, $A_{s}, \Omega_{\nu} h^{2}, \Omega_{m} h^{2}, \Omega_{b} h^{2}, n_{s}, h, w, \tau_{r e}$, and $Y_{p}$. In our calculations, Eqs. (3.1)-(3.4), we assumed a maximum multipole of $l_{\max }=3000$. The relevant experiment specifications are listed in Table 1. Values of the dimensionless bias $\delta_{\lambda} / \sigma_{\lambda}$ are listed in Tables $2 \& 3$ for Planck and a cosmic-variance-limited experiment (CVL), respectively. For each experiment we calculate the bias from filaments and the residual cluster signal, assuming that clusters detected at $5 \sigma$ significance or higher are subtracted out. To estimate the cluster detection significance we consider clusters on the mass-redshift grid M-z. The gas temperature of a given cluster depends on both its redshift and mass assuming it is virialized. In addition, its gas density depends on redshift, and therefore the SZ signal from clusters depend on their masses and redshift regardless of their abundance, which clearly is M-z-dependent. Also, clusters of a given size but at different redshifts subtend different angular scales on the sky, a fact relevant for their detection because of the telescope limited angular resolution.

To assess the signal-to-noise of clusters detection we considered noise contributions from both the primary CMB, detector noise, and point sources. We assume that only data from the 100, 143 and $353 \mathrm{GHz}$ bands is used for cluster detection since lower or higher frequency bands are contaminated by astrophysical foregrounds (More details are given in, e.g. [23]). After removing those 'cells' in M-z space where we expect galaxy clusters to be detected at $5 \sigma$ or higher, we integrate the M-z-deopendent cluster y-parameter over the dark matter halo population, in a procedure described in Eq.(2.12). We assume the Tinker mass function [24]. Finally, to obtain the bias on inferred cosmological parameters in the presence of cluster residuals (i.e. those clusters that have not been detected and masked out), this $\Delta C_{l}$ is used in Eq.(3.3).

In contrast to galaxy clusters, detection and removal techniques cannot be applied to filaments since these irregular low signal structures are not likely to be individually detected, almost certainly not at a sufficiently high level of significance allowing their removal without biasing the CMB sky.

It should be mentioned that our results for the expected bias induced by galaxy clusters (Table 2) are systematically lower than those reported in [19]. A meaningful comparison between the two sets of results cannot be made due to insufficient details on their analysis. A likely source for most of the difference is the overall normalization of the SZ power; while we normalized to the SPT $\left(C_{l=3000}^{S Z}=3.65 \mu K^{2}\right.$ at $150 \mathrm{GHz}$, [25]), their normalization (from Fig.1 in [19]) is considerably higher. Also, different procedures of cluster detection and removal employed in the two works would almost certainly result in different low- and moderate- $l$ power spectrum behavior, this without considering the overall different $l$-dependence expected due to the different forms of the gas density profile and mass function used in the two works.

It is also interesting to note that in the case of Planck the filaments induce bias comparable to the bias induced by galaxy clusters (Table 2), and in the case of the CVL experiment the residual cluster-induced bias is a factor $\sim 2-3$ larger (Table 3 ). This implies that the contribution of filaments to the bias must be accounted for, especially since many more clusters will be detected and removed using non-CMB observations, further boosting the relative 
contribution of filaments-induced anisotropy to the bias.

\section{Discussion}

Our calculations of the filament-induced SZ power spectrum are based on several simplifying assumptions which merit particular attention, given the limited quantitative knowledge of filament structure and physical properties.

The IF gas temperature and density were taken to be uniform, ignoring more likely distributions observed in simulations, with higher temperatures and densities lying along the filament axis and towards nodes, where clusters are thought to reside. However, modeling the distribution of IF gas necessitates a more detailed study of hydrodynamical simulations and a statistical analysis of their results. Our semi-analytic treatment can be readily modified once a more detailed description of IF gas properties is obtained from hydrodynamical simulations. We do not expect a major revision of the results presented here. Note that modification of the IF gas temperature is trivially reflected in the SZ power through its direct dependence on its squared value. Basically, this holds also for the IF electron number density, with the additional effect also on the inferred filament length, as apparent from Eq. (2.3), and consequently also on the shape of the power spectrum, which would peak at higher (lower) multipoles with enhanced (reduced) densities.

Likewise, filament morphology is clearly more complex than plain cylindrical, and is unlikely to be characterized by a single cross section, taken here as the peak distribution value reported in [9]. Filament lengths, too, are unlikely to be determined solely by their masses; a more plausible calculation would assign lengths drawn from a suitable PDF.

Our power spectrum calculations involve the flat sky approximation, which formally fails at angular scales associated with long filaments. In fact, filaments with mass $10^{15} M_{\odot} \cdot h^{-1}$ would be $\sim 140 \mathrm{Mpc} \cdot h^{-1} \mathrm{long}$, and would subtend an angle $\sim 22^{\circ}$ at redshift $z=0.1$. We nonetheless believe that our results are still viable, since the contribution of massive filaments to the SZ power spectrum is quite low due to their extreme rarity, as predicted by the mass function, whereas they would induce the maximal SZ signal at the least probable low inclination angles with the los, for which the flat sky approximation holds. At the largest (and most probable) inclination angle of $90^{\circ}$, the flat sky approximation may seem insufficiently accurate; however, at such angles the optical depth is comparatively small and the SZ signal is relatively weak. Moreover, to obtain a quantitative estimate for the departure of the flatsky from the full-sky calculation at the lowest multipoles, we note that a first order estimate of the accuracy of the approximation could be obtained by realizing that going from flat- to full-sky may be approximated by either taking the limit $l \rightarrow l+\frac{1}{2}$ or $l \rightarrow \sqrt{l(l+1)}[26]$. This implies that by employing the flat-sky approximation we systematically underestimate the filament power spectrum at the lowest multipoles, since the filament power spectrum has a positive slope at $l \lesssim 1000$ (Fig.[2]). Now, beacuase the bias, Eq.(3.3), is proportional to the filament power spectrum this implies that we actually underestimate the level of cosmological parameter bias. Employing these transformations in our calculations shows that the values reported in Tables $2 \& 3$ change only at the third or fourth decimal point. This can be explained by the fact that the flat sky approximation only makes a noticeable difference at very low multipoles. Indeed, we verified that already at $l=10$ there is only $\sim 3-4 \%$ departure, smaller than cosmic-variance at this scale. Compared to other uncertainties in our filament model, the impact of this approximation on our bias estimates is negligibly small. 


\section{Conclusions}

We used a simple model for the intra-filament gas to assess SZ power generated in these large scale structures. We have shown that under certain simplifying assumptions, SZ power induced by filaments could reach a level of $\sim 10 \mu K^{2}$ at multipoles $\ell \sim 1500$ in the RayleighJeans region, with uncertainties directly related to IF gas properties and morphology. SZ power induced by filaments at relevant scales is shown to constitute a non-negligible contribution to the overall SZ signal, with respect to the corresponding power generated by SZ clusters. Unlike clusters, whose SZ signal can be detected and removed from the CMB sky without introducing appreciable bias, the diffuse filament SZ signal cannot be subtracted out. Consequently, the SZ power contributed by filaments can potentially induce a non-negligible bias on several cosmological parameters as shown in Tables $2 \& 3$.

The bias levels shown in Tables 2 \& 3 were obtained from using all 3000 multipoles. We note that by cutting off at lower $l_{\max }$ values the bias decreases. For PLANCK we find that already at $l_{c} \approx 1000$ both $A_{s}, n_{s}$ and $w$ are biased by a level comparable to the nominal statistical uncertainty. With the CVL experiment this critical multipole number is pushed towards $\approx 1500$.

It is interesting to note that the pervasive web of filamentary structure represents the most extended screen that sets a lower limit to the degree of Comptonization of the CMB, a limit that cannot be removed when attempting to use spectral distortions of the CMB as cosmological probes of energy release processes in the early universe. From the filamentinduced temperature anisotropy power at the lowest 2000-3000 multipoles we estimate an rms comptonization parameter $y_{r m s} \sim 7 \times 10^{-7}$. This value scales approximately as $\propto$ $\left(n_{e} T_{e}\right)^{2}$. Due to modeling uncertainties - e.g. filament morphology, filament mass function, gas density and temperature - there clearly is an appreciable uncertainty in our estimate for

$y_{r m s}$. However, since the missing baryons are likely to be accounted for by warm IF gas, $n_{e}$ is not expected to significantly deviate from the fiducial value adopted here because of the constraint set by the requirement that the missing baryons provide $30-40 \%$ of the total baryon budget. Ultimately, these uncertainties will be lowered through more realistic high resolution hydrodynamical simulations.

\section{Acknowledgments}

This work was supported by a US-IL Binational Science Foundation grant 2008452, and by the James B. Ax Family Foundation. We thank two anonymous referees for useful comments. Use of CAMB for calculations of primary CMB power spectra is acknowledged. 


\begin{tabular}{|c|c|c|c|c|}
\hline Experiment & $f_{\text {sky }}$ & $\nu[G H z]$ & $\theta_{b}\left[1^{\prime}\right]$ & $\Delta_{T}[\mu K]$ \\
\hline & & 30 & 33 & 4.4 \\
& & 44 & 23 & 6.5 \\
& & 70 & 14 & 9.8 \\
Planck & \multirow{3}{*}{0.65} & 100 & 9.5 & 6.8 \\
& & 143 & 7.1 & 6.0 \\
& & 217 & 5.0 & 13.1 \\
& & 543 & 5.0 & 40.1 \\
& & 545 & 5.0 & 401 \\
& & 5.0 & 18300 \\
\hline \hline
\end{tabular}

Table 1. Sensitivity parameters for Planck. The CVL experiment is noiseless. The beam full width half maximum (FWHM) resolution $\theta_{b}$ is related to the gaussian beamwidth via $\theta_{b}=\sqrt{8 \ln (2)} \theta_{b}$ and the detector noise power spectrum is $C_{l}^{T, \text { det }}=\left(\Delta_{T} \theta_{b}\right)^{2} e^{l^{2} \sigma_{b}^{2}}$. The figures quoted here assume 1 year of observation [27].

\begin{tabular}{|c|c|c|c|c|c|c|c|c|c|}
\hline Foreground & $A_{s}$ & $\Omega_{\nu} h^{2}$ & $\Omega_{m} h^{2}$ & $\Omega_{b} h^{2}$ & $n_{s}$ & $h$ & $w$ & $\tau$ & $Y_{p}$ \\
\hline Filaments & 1.5 & -0.8 & -0.3 & 0.0 & 1.8 & -0.8 & -1.3 & -0.6 & -0.3 \\
\hline Cluster Residuals & 1.1 & -0.8 & -0.3 & 0.1 & 1.4 & -0.7 & -1.1 & -0.4 & -0.3 \\
\hline
\end{tabular}

Table 2. Expected bias of the cosmological model due to an unaccounted-for filament or cluster contribution to the CMB power spectrum measured by Planck in units of the nominal statistical uncertainty $\sigma_{\lambda}$. In the case of filaments the assumed electron number density and gas temperature are $n_{0}=4.3 \times 10^{-6} \mathrm{~cm}^{-3}$ and $k T_{e}=1 \mathrm{keV}$, respectively. The first row shows the bias due to CMB comptonization by the warm plasma in filaments. In the second row we present the effect of the thermal SZ effect from galaxy clusters. Shown is the reduced bias level that would be obtained if the clusters detected by Planck would be removed from the CMB sky.

\begin{tabular}{|c|c|c|c|c|c|c|c|c|c|}
\hline Foreground & $A_{s}$ & $\Omega_{\nu} h^{2}$ & $\Omega_{m} h^{2}$ & $\Omega_{b} h^{2}$ & $n_{s}$ & $h$ & $w$ & $\tau$ & $Y_{p}$ \\
\hline Filaments & 5.6 & -7.5 & -1.9 & 0.7 & 6.5 & -6.2 & -8.4 & -2.6 & -1.6 \\
\hline Cluster Residuals & 13.4 & -17.6 & -4.4 & 1.7 & 13.7 & -11.7 & -15.4 & -5.2 & -4.0 \\
\hline
\end{tabular}

Table 3. Same as Table 2, but for a CVL experiment

\section{References}

[1] R. Cen, and J.P. Ostriker J.P., Where Are the Baryons?, ApJ 514 (1999) 1-6, [astro-ph/9806281].

[2] E.J. Hallman, B.W. O'Shea, J.O. Burns, M.L. Norman, R. Harkness, R. Wagner, The Santa Fe Light Cone Simulation Project. I. Confusion and the Warm-Hot Intergalactic Medium in Upcoming Sunyaev-Zel'dovich Effect Surveys, ApJ 671 (2007) 27-39, [arXiv:0704.2607].

[3] R. Davé, R. Cen, J.P. Ostriker, et al., Baryons in the Warm-Hot Intergalactic Medium, 552 (2001) 473-483, [astro-ph/0007217].

[4] R. Fujimoto. et al., Probing Warm-Hot Intergalactic Medium Associated with the Virgo Cluster using an Oxygen Absorption Line, Pub. Astron. Soc. Japan 56 (2004) 29-34, [astro-ph/0409010].

[5] Y. Takei, J.P Henry, A. Finoguenov, K. Mitsuda, T. Tamura, R. Fujimoto, U.G. Briel, Warm-Hot Intergalactic Medium Associated with the Coma Cluster, ApJ 655 (2007) 831-842, [astro-ph/0610424]. 
[6] A. Kull, H. Bohringer, Detection of filamentary X-ray structure in the core of the Shapley supercluster, Astron. Astrophys. 341 (1999) 23-28, [astro-ph/9812319].

[7] L. Zappacosta, F. Mannucci, R. Maiolino, R. Gilli, A. Ferrara, A. Finoguenov, N.M. Nagar, \& D.J. Axon, Warm-hot intergalactic baryons revealed, Astron. Astrophys. 394 (2002) 7-15, [astro-ph/0208033].

[8] A. Finoguenov, U.G. Briel, J.P. Henry, XMM-Newton discovery of an X-ray filament in Coma, Astron. Astrophys. 410 (2003) 777-784, [astro-ph/0309019].

[9] J.M. Colberg, K.S. Krughoff, A.J. Connolly, Inter-cluster filaments in a CDM Universe, MNRAS 359 (2005) 272-282, [astro-ph/0406665].

[10] Y. Noh, J.D. Cohn, The geometry of the filamentary environment of galaxy clusters, MNRAS 413 (2011) 301-312, [arXiv: 1011.1000].

[11] J.S. Klar, J.P. Mücket, Filaments and sheets of the warm-hot intergalactic medium, MNRAS 423 (2012) 304-319, [arXiv: 1203.1800].

[12] A. Cooray, Large scale pressure fluctuations and the Sunyaev-Zel'dovich effect, Phys. Rev. D. 62 (2000) 103506, [astro-ph/0005287].

[13] E. Komatsu, U. Seljak, The Sunyaev-Zel'dovich angular power spectrum as a probe of cosmological parameters, MNRAS 336 (2002) 1256-1270, [astro-ph/0205468].

[14] A. Refregier, R. Teyssier, Numerical and analytical predictions for the large-scale Sunyaev-Zel'dovich effect, Phys. Rev. D. 66 (2002) 043002, [astro-ph/0012086].

[15] F. Atrio-Barandela, J.P. Mücket, The Contribution of the Intergalactic Medium to Cosmic Microwave Background Anisotropies, ApJ 643 (2006) 1-7, [astro-ph/0601424].

[16] S. Zaroubi, G. Suiqres, G. De Gaspreis., A.E. Evrard, Y. Hoffman, J. Silk, Deprojection of Galaxy Cluster X-Ray, Sunyaev-Zeldovich Temperature Decrement, and Weak-Lensing Mass Maps, ApJ 561 (2001) 600-620, [astro-ph/0010508].

[17] S. Doddelson, (2003), Modern Cosmology, Academic Press,

[18] J. Shen, T. Abel, H.J. Mo, R.K. Sheth, An Excursion Set Model of the Cosmic Web: The Abundance of Sheets, Filaments, and Halos, ApJ 645 (2006) 783-791, [astro-ph/0511365].

[19] N. Taburet, N. Aghanim, M. Douspis, \& M. Langer, Biases on the cosmological parameters and thermal Sunyaev-Zel'dovich residuals, MNRAS 392 (2009) 1153-1158, [astro-ph/0809.1364].

[20] M. Tegmark, \& G. Efstathiou, A method for subtracting foregrounds from multifrequency CMB sky maps, MNRAS 281 (1996) 1297-1314, [astro-ph/9507009].

[21] M. Tegmark, A. de Oliveira-Costa, \& A.J. Hamilton, High resolution foreground cleaned CMB map from WMAP, Phys. Rev. D. 68 (2003) 123523, [astro-ph/0302496].

[22] D. Larson et al., Seven-year Wilkinson Microwave Anisotropy Probe (WMAP) Observations: Power Spectra and WMAP-derived Parameters, Ap. J. Supp. 192 (2011) 16, [arXiv: 1001.4635$]$.

[23] M. Shimon, S. Sadeh, \& Y. Rephaeli, Neutrino mass inference from Sunyaev-Zel'dovich surveys, MNRAS 412 (2011) 1895-1904, [arXiv:1009.4110].

[24] Tinker, J., Kravtsov, A. V., Klypin, A., et al. Toward a halo mass function for precision cosmology: the limits of universality, ApJ 688 (2008) 709, [arXiv:0803.2706].

[25] C. L. Reichardt, L. Shaw, O. Zahn, et al. A measurement of secondary cosmic microwave background anisotropies with two years of South Pole Telescope observations [arXiv: 1111.0932].

[26] Bernardeau, F., Pitrou, C., \& Uzan, J.-P., CMB spectra and bispectra calculations: making the flat-sky approximation rigorous, JCAP 2 (2011) 15, [arXiv:1012.2652]. 
[27] http://www.rssd.esa.int/index.php?project=Planck 